\title{
Robotic versus other nephroureterectomy techniques: a systematic review and meta-analysis of over 87,000 cases
}

\author{
Alessandro Veccia ${ }^{1,2} \cdot$ Alessandro Antonelli $^{2} \cdot$ Simone Francavilla ${ }^{2} \cdot$ Claudio Simeone $^{2} \cdot$ Georgi Guruli $^{1}$. \\ Homayoun Zargar ${ }^{3}$. Sisto Perdoná ${ }^{4} \cdot$ Matteo Ferro $^{5}$. Giuseppe Carrieri ${ }^{6}$. Lance J. Hampton ${ }^{1}$. Francesco Porpiglia ${ }^{7}$. \\ Riccardo Autorino ${ }^{1}$ [i]
}

Received: 9 June 2019 / Accepted: 10 November 2019

(c) Springer-Verlag GmbH Germany, part of Springer Nature 2019

\begin{abstract}
Purpose To perform a systematic review and meta-analysis of the literature inherent robotic nephroureterectomy (RNU) and to compare its outcomes with those of other nephroureterectomy (NU) techniques.

Methods A systematic literature search was performed up to April 2019 using PubMed, Embase ${ }^{\circledR}$, and Web of Science. The Preferred Reporting Items for Systematic Review and Meta-analysis Statement was followed for study selection. The following data were extracted for each study: baseline features, surgical outcomes, oncological outcomes, and survival outcomes. Stata 15.0 was used for statistical analysis.

Results Literature search identified 80 studies eligible for the meta-analysis and overall 87,291 patients were included in the analysis: open NU (ONU; $n=45,601)$, hand-assisted laparoscopic NU (HALNU; $n=442$ ), laparoscopic NU (LNU $n=31,093)$, and RNU $(n=10,155)$. RNU was more likely to be performed in those patients with multifocal tumor location (proportion: $0.19 ; 95 \%$ CI 0.14, 0.24) and high-grade disease (proportion: 0.70; 95\% CI 0.53, 0.68). The lowest EBL was recorded in the RNU group (weighted mean (WM) $163.31 \mathrm{~mL} ; 95 \%$ CI 88.94, 237.68), whereas the highest was in the ONU group $(414.99 \mathrm{~mL} ; 95 \%$ CI 378.52, 451.46). Operative time was shorter for ONU (224.98 mL; 95\% CI 212.26, 237.69). RNU had lower rate of intraoperative complications $(0.02 ; 95 \%$ CI $0.01,0.05)$. ONU showed higher odds of transfusions $(0.20$; $95 \%$ CI 0.15, 0.25). LOS was statistically significantly shorter for the RNU group (5.35 days; 95\% CI 4.97, 5.82). HALNU seemed to present lower risk of PSM $(0.02 ; 95 \%$ CI $-0.01,0.05)$, and lower risk of recurrence $(0.22 ; 95 \%$ CI $0.15,0.30)$, metastasis $(0.07 ; 95 \%$ CI $0.05,0.10)$, and cancer-related death $(0.03 ; 95 \%$ CI $0.01,0.06)$. ONU showed the lowest 5 years cancer specific survival (proportion: $0.77 ; 95 \%$ CI $0.74,0.80$ ). No correlation was found between the surgical technique and recurrence-free and cancer-specific survival.

Conclusions Evidence regarding RNU for the treatment of UTUC is increasing but it remains quite sparse and of low quality. Despite this, RNU seems to be safe, and to offer the advantages of a minimally invasive approach without impairing the oncological outcomes. Nevertheless, ONU, HALNU, and LNU still represent a valid, and commonly used surgical treatment option. As RNU becomes more popular, and concerns related to its use remain, the best surgical technique for NU remains to be determined.
\end{abstract}

Keywords Open radical nephroureterectomy $\cdot$ Hand-assisted radical nephroureterectomy $\cdot$ Laparoscopic radical nephroureterectomy $\cdot$ Robotic radical nephroureterectomy $\cdot$ Upper tract urothelial carcinoma

\section{Introduction}

Electronic supplementary material The online version of this article (https://doi.org/10.1007/s00345-019-03020-1) contains supplementary material, which is available to authorized users.

Riccardo Autorino

ricautor@gmail.com

Extended author information available on the last page of the article
Current guidelines recommend radical nephroureterectomy (NU) with bladder cuff excision (BCE) as the standard treatment for high-risk upper tract urothelial carcinoma (UTUC) [1]. The last two decades witnessed the introduction and diffusion of minimally invasive surgery techniques for NU [2]. Laparoscopic NU (LNU) showed to offer lower surgical 
morbidity and shorter length of hospital stay, despite controversial findings about its oncological safety [3]. Equally, robotic NU (RNU) has been explored and increasingly implemented as robotic technology can facilitate key steps of the NU procedure, such as BCE and lymphadenectomy $[3,4]$. However, current literature still lacks robust evidence regarding RNU outcomes, as available data are mostly from small series or population-based studies [5-7].

Aim of this study is to perform a systematic review and meta-analysis of the literature inherent RNU and to compare its outcomes with those of other NU techniques, including open NU (ONU), hand-assisted laparoscopic nephroureterectomy (HALNU) and LNU.

\section{Methods}

\section{Literature research strategy}

Two different authors (A.V. and S.F.) independently screened literature regarding ONU, HALNU, LNU, and RNU. The results were assessed by a third author (R.A.). PubMed, Embase ${ }^{\circledR}$, and Web of Science were queried up to April 2019. A free-text strategy was deemed to be the most suitable for this topic (as summarized in supplementary material 1). Comparative and non-comparative retrospective, prospective, non- and randomized studies were included.

Non-English language articles, conference abstract, conference paper, reviews, letters, notes, editorials, and book chapters and studies published before 2000 were excluded. Possible missing articles were retrieved by the assessment of the reference list of each article included.

\section{Studies selection}

The Preferred Reporting Items for Systematic Review and Meta-analysis Statement (PRISMA Statement-www.prism a-statement.org) $[8,9]$ was followed for study selection. Eligible studies were identified based on the following inclusion criteria: studies on minimally invasive NU, in adult population (patients older than 18 years old), and for diagnosis of UTUC. Given the very limited number of studies, it was established to not include in the analysis data about laparoendoscopic single site NU (LESS-NU), and laparoendoscopic single site robotic nephroureterectomy (LESS-RNU). After a first screening based on title and abstract, full texts of potentially eligible studies were evaluated, and those meeting inclusion criteria were selected.

\section{Data extraction}

The following data were extracted for each study:
- baseline features: age, gender $(\widehat{\jmath})$, body mass index (BMI), race (Caucasian), Charlson Comorbidity Index $(\mathrm{CCI}) \geq 2$, American Society of Anesthesiologist (ASA) score $\geq 3$, tumor location (pelvicalyceal, ureteral, multifocal), and surgery performed in an academic hospital;

- surgical outcomes: estimated blood loss (EBL), operative time (OT), intraoperative complications, transfusions, overall complications, major complications (Clavien $\geq 3$ ), and length of stay;

- pathological outcomes: $\mathrm{pT} \geq 3$, high-grade tumor, $\mathrm{pN}+$, nodes removed, and positive surgical margins (PSM);

- survival outcomes: recurrence, metastasis, death, 2- and 5-years recurrence free survival (RFS), 2- and 5-years cancer specific survival (CSS), and correlation between surgical technique and RFS and CSS.

\section{Study quality assessment}

Level of Evidence (LE) of the studies was established according to the Oxford Level of Evidence Working Group 2011 [10]. The quality of the reports was stratified conforming to the Newcastle-Ottawa Assessment Scale (NOS) for non-randomized controlled trials [11]. A score of 5 was considered low, 6-7 intermediate, and 8-9 high. Jadad Scale was adopted as evaluation mean for randomized controlled trials (RCT's) [12]. A score of 2 or less identified lowquality studies, 3 intermediate quality reports, and $4-5$ high quality RCT.

\section{Data analysis}

Data were collected into separate datasheets per each outcome assessed. Variables reported as median (range) were converted to mean \pm standard deviation (SD) through a dedicated formula [13]. Grand mean was calculated for those data inherent the same variable but split in two groups. Mean \pm SD were transformed into mean (95\% confidence interval) (CI). It was performed pooled analysis of mean (95\% CI) for continuous values using the metan function. For dichotomous values, cumulative analysis of proportion was performed using the metaprop function. Regarding survival outcomes, log of Hazard Ratios (HR) and 95\% CI were generated to pool the data. Heterogeneity among the studies was weighted according to random effect [14]. Funnel plots were evaluated to establish the presence of publication bias.

We also performed a sensitivity analysis of survival outcomes of those patients who underwent NU with purely open or intracorporeal BCE. Stata ${ }^{\circledR} 15.0$ (StataCorp 2017. Stata Statistical Software: release 15. StataCorp LLC, College Station, TX, USA) was used as software for statistical analysis. Statistical significance was set at $p \leq 0.05$. 


\section{Results}

\section{Studies features}

The PRISMA flow chart is showed in Fig. 1. The literature search identified 80 studies eligible for the metaanalysis [1-80] (supplementary table 1). Over the last decade, there has been an increase in number of studies on RNU (from $0 \%$ of studies in 2000-2009 to 36\% in the period 2010-2019) (supplementary Fig. 1). Two studies were prospective LE 2 [4, 14], and one RCT LE 1 [35]. The remaining studies were all retrospective of LE 3 . Twenty reports were of high quality $[1,2-15,16-18$, 19-21-23-28-31-33-37-39, 40-43-45-51-78-79, 80], and the only one RCT was of quality 3 [35]. The remaining were of intermediate quality. Overall, 87,291 patients were included in the analysis of which 45,601 ONU, 442 HALNU, 31,093 LNU, and 10,155 RNU.

\section{Baseline features}

RNU was more likely to be performed in those patients with multifocal tumor location (proportion: $0.19 ; 95 \% \mathrm{CI}$ $0.14,0.24)$. There was no statistically significant difference among the surgical techniques regarding other baseline parameters (supplementary material 2).
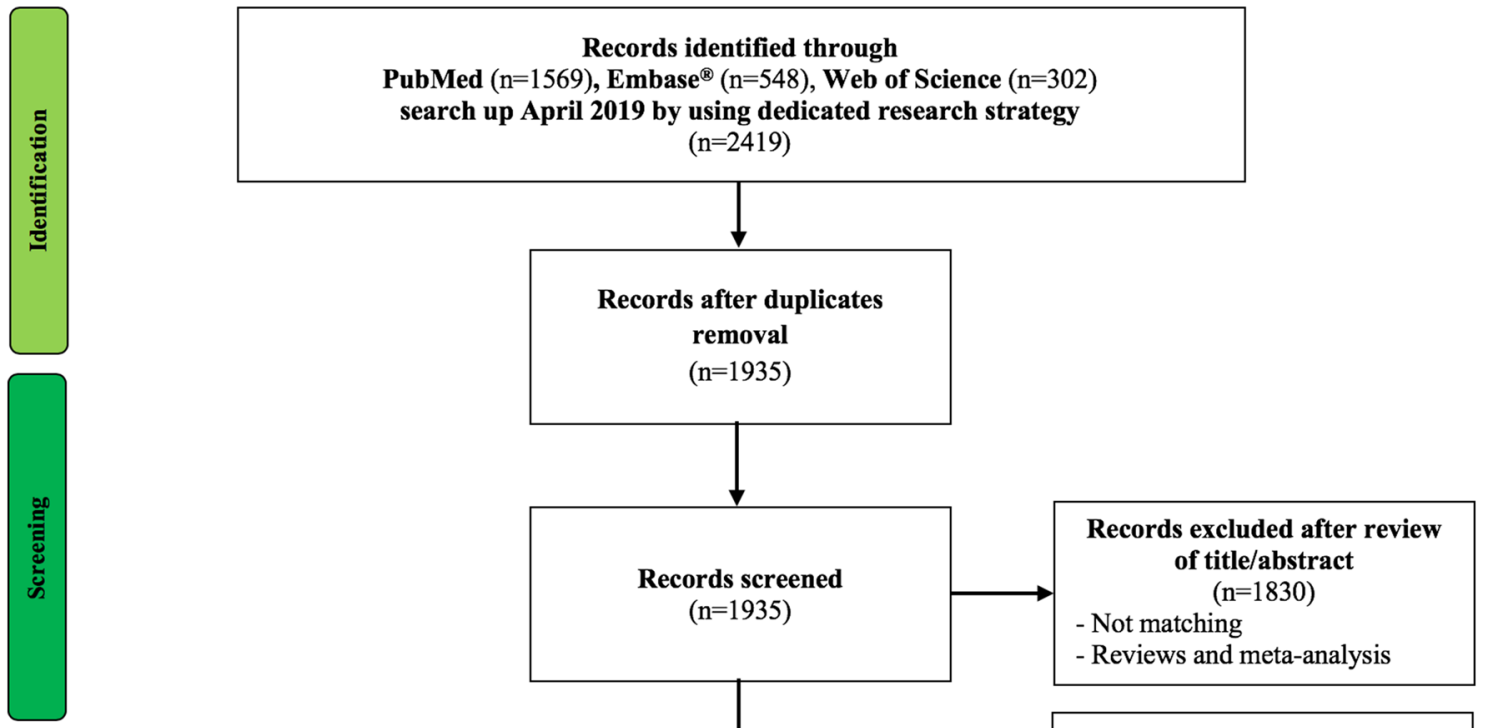

removal
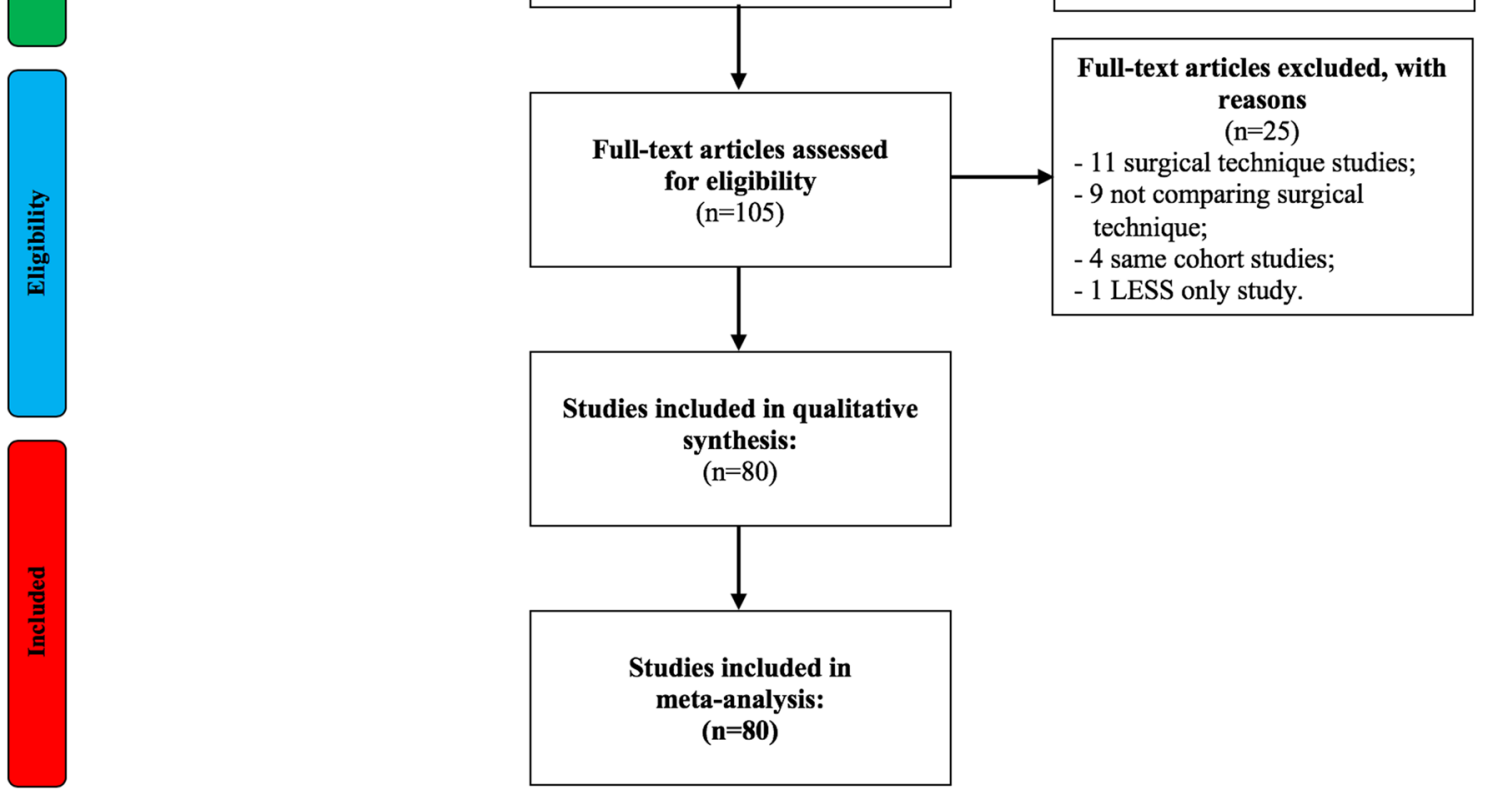

Studies included in qualitative synthesis: $(\mathrm{n}=80)$

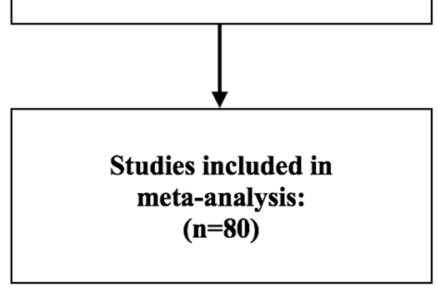

Fig. 1 PRISMA flow chart 


\section{Surgical outcomes}

Intracorporeal $\mathrm{BCE}$ was performed for all RNU procedures, while only in $50 \%$ of the LNU procedures $(95 \%$ CI $0.31,0.70)$. The lowest EBL was recorded in the RNU group (weighted mean (WM) $163.31 \mathrm{~mL} ; 95 \%$ CI 88.94, 237.68), whereas the highest was in the ONU group (WM $414.99 \mathrm{~mL} ; 95 \%$ CI 378.52, 451.46). Operative time was shorter for ONU (WM $224.98 \mathrm{~mL}$; 95\% CI 212.26, 237.69), while there was no statistically significant difference for the other procedures.

RNU had the lowest rate of intraoperative complications (proportion: $0.02 ; 95 \%$ CI 0.01, 0.05). There was no statistically significant difference in terms of transfusion rate between RNU and LNU (10\% and 7\%, respectively). Instead, ONU showed the highest odds of transfusions (proportion: $0.20 ; 95 \%$ CI $0.15,0.25$ ). We did not see differences in terms of overall- and major complications. LOS was statistically significantly shorter for the RNU group (WMD 5.35 days; 95\% CI 4.97, 5.82), whereas ONU presented the longest LOS (WMD 10.27; 95\% CI 10.27 days; 8.86; 11.68) (supplementary material 2 ).

\section{Pathological outcomes}

RNU was performed more in those patients with high-grade pathology (proportion: 0.70; 95\% CI 0.53, 0.68), while we did not find any statistically significant difference between ONU, HALNU, and LNU (50\%, 57\%, and 50\% probability, respectively). There was no statistically significant difference among the procedures regarding $\mathrm{pN}+$, as well as of lymph nodes removed too. HALN presented the lowest risk of PSM (proportion: 0.02; 95\% CI - 0.01, 0.05) (supplementary material 2).

\section{Survival outcomes}

Overall, HALNU showed the lowest risk of recurrence (proportion: 0.22 ; 95\% CI 0.15, 0.30), metastasis (proportion: $0.07 ; 95 \%$ CI $0.05,0.10$ ), and cancer-related death (proportion: $0.03 ; 95 \%$ CI 0.01, 0.06). ONU showed the lowest 5-year cancer specific survival (CSS) (proportion:
$0.77 ; 95 \%$ CI $0.74,0.80)$, whereas there was no statistically significant difference among HALNU, LNU, and RNU in terms of 2- and 5-year recurrence free survival (RFS) and cancer specific survival (CSS) (supplementary material 2). We did not find correlation between the surgical technique (ONU vs LNU vs RNU) and RFS and CSS, but only one study assessed these parameters for RNU [79] (Fig. 2 and supplementary material 2 ).

\section{Sensitivity analysis of survival outcomes of open vs intracorporeal BCE}

Intracorporeal BCE showed lower rate of bladder recurrence (proportion: 0.18 ; 95\% CI 0.12, 0.24 vs proportion: 0.27 ; 95\% CI 0.20, 0.33). Despite patients with intracorporeal $\mathrm{BCE}$ being more likely to develop postoperative metastasis, the difference with the open BCE group was not statistically significant ( $18 \%$ vs $12 \%$; $p=0.059)$. Moreover, no statistically significant difference was found in terms of recurrence and death (Fig. 3).

\section{Bias assessment}

The evaluation of the funnel plots of each outcome demonstrated a quite diffused presence of bias among the studies included (supplementary Fig. 2).

\section{Discussion}

To the best of our knowledge, herein we present the data of the largest systematic review and meta-analysis on NU to date, including 80 studies and a cohort of about 87,000 patients. Our analysis raises some points which are worth discussing.

Patients showed no statistically significant difference in terms of baseline features, and there was a prevalence of Caucasian male subjects with a mean age of 67.6 years. These figures mirror those from well-known epidemiological data about UTUC [15]. Less forecasted was the higher proportion of multifocal tumor in the RNU group compared to the others (19\% vs overall $10 \%$ ). Nevertheless, none of the
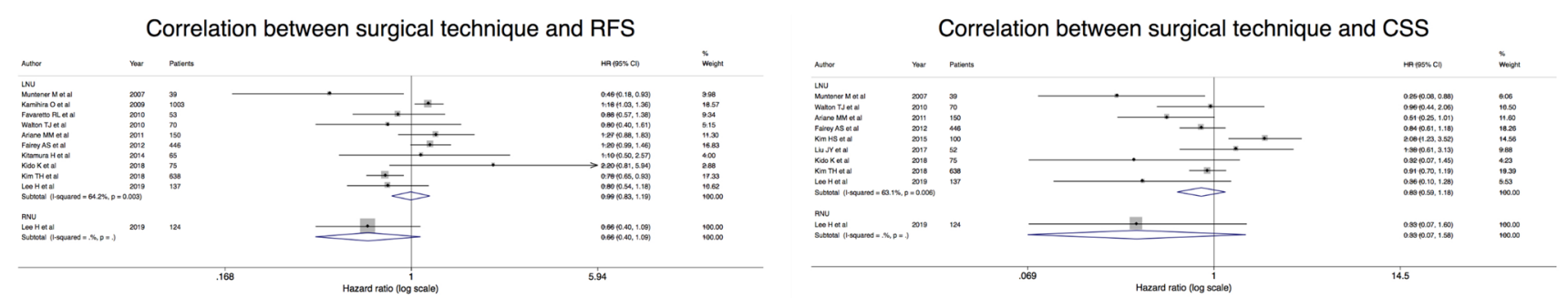

Fig. 2 Correlation between surgical technique and survival outcomes 

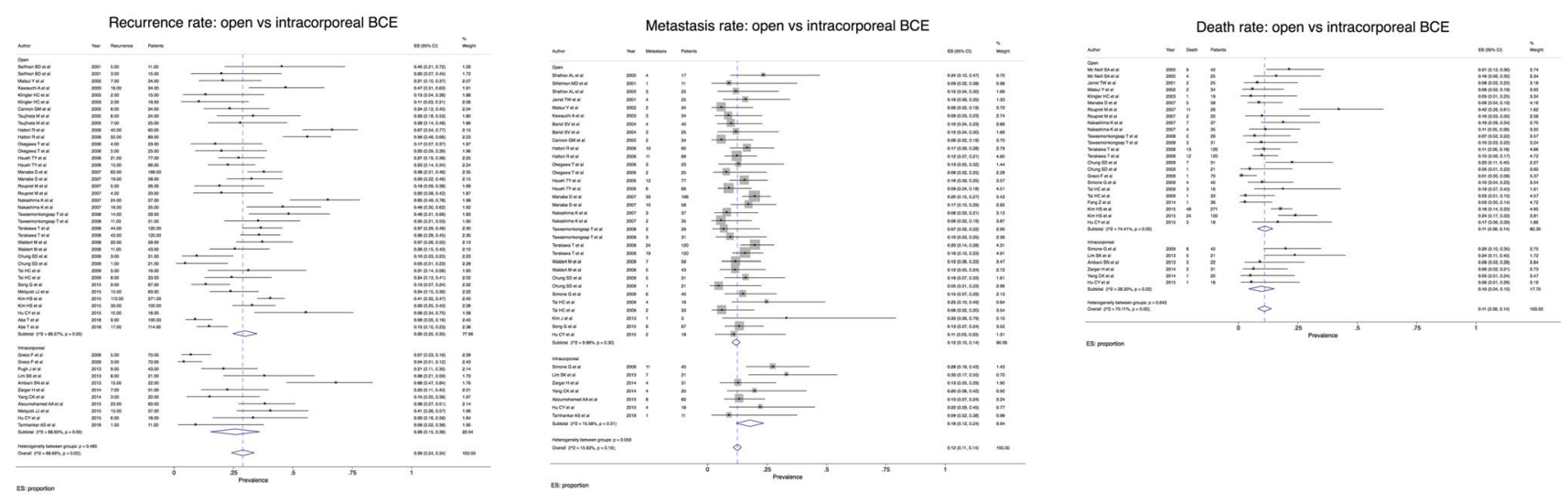

Fig. 3 Comparison survival outcomes of open vs intracorporeal bladder cuff excision

studies reported significative difference in terms of tumor location.

Regarding surgical outcomes, RNU showed the lowest EBL compared to other surgical procedures. This finding is consistent with a recent report assessing a cohort of 422 patients who underwent ONU, LNU, and RNU, demonstrating lower EBL for the robotic group [16]. Lower EBL translated into a lower transfusion rate, whereas ONU showed higher odds of transfusions (20\%) [17]. LNU also had higher transfusion rate, but it was not clinically significant compared to RNU (11\% vs 9\%). A previous study comparing RNU and LNU demonstrated a more significant difference (8\% and $30 \%$, respectively) [18].

Differently from the other reports on this topic, RNU showed no statistically significant difference in OT compared to the other procedures. Melquist et al. evaluated LNU and RNU and the latter demonstrated a longer OT [18]. In addition, in our series ONU registered the shortest OT. Despite this, the difference with the robotic one was of only 26 min which could be a consequence of the robot docking or repositioning during the procedure. In fact, a recent comparative study on RNU performed with Xi platform demonstrated OT to be shorter of about $50 \mathrm{~min}$ [19], as the advantage of this new platform is to smoothly perform the entire procedures without need for re-docking or patient repositioning [20]. Unfortunately, there were no other studies available to perform a sensitivity analysis on this point.

RNU demonstrated a slightly lower rate of intraoperative complications ( $2 \%$ vs overall $4 \%$ ). A reasonable explanation might reside in the advantages provided by the robotic surgery such as better vision, and instruments precision, maneuverability and delicacy in applying traction on the tissues [6]. On the other hand, overall- and major complications were overlapping between RNU and the other ones. A population-based study evaluated the complications rate among ONU, LNU, and RNU within a population of about 20,000 patients and found no-difference in terms of 90-day mortality and complications [21]. Notwithstanding this, in our cohort RNU showed a smaller, better clinical trend of complications with a $17 \%$ vs $20 \%$ of the ONU and LNU. Though complications were overlapping, RNU had a significantly shorter LOS. This data mirrored those from previous studies [16]. Indeed, Trudeadu et al. conducted a propensity score matched paired analysis between LNU and RNU within the Nationwide Impatient Sample database. The authors concluded that there was no-difference regarding LOS among the two procedures [22]. Notably, our results pointed out shorter LOS for RNU compared to LNU too. This could be explained with the increase of the expertise and diffusion of the robotic procedure which drastically improved the outcomes. Lee et al. found that after the first 42 robotic procedures there was a reduction of EBL, LOS, and analgesic usage [16]. However, differences in practice patterns in different countries could also account for this finding, as in the US, where most robotic series come from, there is a general trend to discharge home earlier compared to European and Asian countries.

The assessment of pathological outcomes proved no statistically significant difference among all the procedures about the pathological stage ( $\mathrm{pT} \geq 3$ and $\mathrm{N}+$ ), while patients with high-grade tumor were more likely to undergo RNU. This finding seems to reflect more on a general trend in favor of RNU during the last decade than a specific choice of robotic surgery for this subset of patients. Several studies underlined this point highlighting the dissemination of robotics and the decrease of the ONU and LNU [21,23].

In our study, RNU showed to yield higher number of nodes with about 11 nodes removed per procedure against 7 of ONU and LNU, and 5 of HALNU. Zargar et al. in one of the first experience on RNU already demonstrated the advantages of robotics during lymph node dissection reporting a mean value of 9.4 harvested nodes [24]. Compared to ours, also Moschini et al. achieved a similar value of nodes resected during ONU and LNU [25]. Nevertheless, the 
number of lymph nodes yielded was reported in a low number of studies with a high heterogeneity among the studies $\left(I^{2}=99 \%\right)$. Thus, this data should be interpreted with caution considering the several bias which might have affected this finding such as selection bias, different experience, and hospital volume.

A lower incidence of recurrence, metastasis, and cancerrelated death was found for HALNU, and these findings were consistent for most studies on HALNU. On the other hand, ONU, LNU, and RNU studies were characterized by some degree of heterogeneity. Two-year RFS and CSS were overlapping among all the procedures, while we found a lower 5 -year CSS in the ONU group. This data was different from previous literature. Indeed, Nouralizadeh et al. performed a literature review on ONU, HALNU, and LNU, and none of the procedures showed to overcome the others regarding survival outcomes [26]. This inconsistency might be a consequence of the larger number of studies and sample size of our analysis (80 vs 52 studies and 87,291 vs 19,195 patients). In addition, our meta-analysis is the first to include RNU which could have influenced the results of the overall cumulative analysis.

One of the main concerns regarding minimally invasive $\mathrm{NU}$ is the risk of recurrence and tumor seeding, including bladder recurrence $[27,28]$. A recent meta-analysis on the comparison between ONU and LNU suggested poorer outcomes for the latter [29]. Simone et al. achieved the same results in the only RCT available, but the sample size did not allow to generalize its findings [30]. Our sensitivity analysis showed no statistically significant difference among open and intracorporeal BCE in terms of recurrence, and death, but this was not the case regarding bladder recurrence. In fact, we found lower rate of bladder recurrence in the intracorporeal BCE group. Most of the data regarding intracorporeal BCE come from studies assessing RNU and the lower rate of bladder recurrence could be due to the advantages of the robotic to manage the distal ureter [6]. Of note, most of the studies included mixed techniques to approach bladder cuff, so fewer studies addressed specifically a BCE management. Thus, a low number of studies could be pooled explaining the fickle approach to BCE.

To note, it was not possible to account for those patients who underwent adjuvant bladder instillation after the procedure, even though recent evidence suggests no advantage in terms of survival outcomes [31]. On the contrary, we found higher risk of metastasis in the intracorporeal group, which however could be related to the fact that the RNU group presented more multifocal and higher-grade disease. Nevertheless, this finding should be further scrutinized.

This meta-analysis is supported by its adequate and rigorous methodological approach, and the large sample size. Notwithstanding these, our study presents some intrinsic limitations that should be disclosed. This is a meta-analysis of retrospective and non-randomized studies which are affected by several bias which cannot be avoided. The retrospective nature of the studies includes selection, attrition, reporting and other bias which might have influenced the results. Indeed, it was not possible to establish the blinding of participants or personnel, the blinding of outcomes assessment, and in some cases incomplete outcomes assessment was noticed. Nevertheless, the inclusion of prospective and RCTs could have balanced our findings. Second, most of the reports are based on data of NUs performed with several techniques of BCE, whose oncological impact remains unclear. Unfortunately, we could not perform a specific analysis for the myriad of BCE techniques, such as pluck technique, ureter stripping, transurethral resection, or intussusception. Third, the different studies were from different institutions and set in different countries, so the nonclinical factors might have had an impact on outcomes. This could also be in-part responsible of the high heterogeneity registered for most of the studies included. Fourth, the study distribution is unbalanced in favor of ONU, and LNU, so the data on the other techniques might be weak. Fifth, this analysis includes comparative and non-comparative studies. Comparative studies are related to similar conditions among the arms and this could have influenced the results in favor of one arm rather than another. For this reason our results should be interpreted cautiously. Last, the sample size of most of the studies is small, thus they might be underpowered to evaluate the outcomes assessed. Furthermore, the large sample size reports are few and in many cases population-based. Another limitation that should be acknowledged is the low number of studies about RNU. Given these facts, our data should be interpreted by taking these limitations into account. Moreover, our analysis did not consider other kidney sparing procedures for the management of UTUC [32].

\section{Conclusions}

Evidence regarding RNU for the treatment of UTUC is increasing but it remains quite sparse and of low quality. Despite this, RNU seems to be safe, and to offer the advantages of a minimally invasive approach without impairing the oncological outcomes. Nevertheless, ONU, HALNU, and LNU still represent a valid, and commonly used surgical treatment option. As RNU becomes more popular, and concerns related to its use remain, the best surgical technique for NU remains to be determined. Future clinical research should ideally aim at prospective randomized studies, possible on multicenter scale.

Acknowledgements Dr. Alessandro Veccia is an Italian Society of Urology-American Urological Association (SIU-AUA) research 
fellow. Funding for his fellowship is also provided by the VCU Urology Research Fund.

Author contributions Protocol/project development: AV, RA. Data collection or management: AV, SF. Data analysis: AV, RA. Manuscript writing / editing: AV, RA, AA, and HZ. Other (Supervision): CS, GG, SP, MF, GC, LJH, FP.

Funding: None.

\section{Compliance with ethical standards}

Conflict of interest: The authors declare that they have no conflict of interest.

Research involving Human Participants and/or Animals This article does not contain any studies with human participants or animals performed by any of the authors.

Informed consent Not applicable.

\section{References}

1. Rouprêt M, Babjuk M, Compérat E, Zigeuner R, Sylvester RJ, Burger M, Cowan NC, Böhle A, Van Rhijn BW, Kaasinen E, Palou J, Shariat SF (2015) European Association of Urology Guidelines on upper urinary tract urothelial cell carcinoma: 2015 update. Eur Urol 68:868-879

2. Mullen E, Ahmed K, Challacombe B (2017) Systematic review of open versus laparoscopic versus robot-assisted nephroureterectomy. Rev Urol 19:32-43

3. Gakis G, Schubert T, Alemozaffar M, Bellmunt J, Bochner BH, Boorjian SA, Daneshmand S, Huang WC, Kondo T, Konety BR, Laguna MP, Matin SF, Siefker-Radtke AO, Shariat SF, Stenzl A (2017) Update of the ICUD-SIU consultation on upper tract urothelial carcinoma 2016: treatment of localized high-risk disease. World J Urol 35:327-335

4. Lee SM, McKay A, Grimes N, Umez-Eronini N, Aboumarzouk OM (2019) Distal ureter management during nephroureterectomy: evidence from a systematic review and cumulative analysis. J Endourol 33:263-273. https://doi.org/10.1089/end.2018.0819

5. Hemal A, Stansel I, Babber P, Patel M (2011) Robotic-assisted nephroureterectomy and bladder cuff excision without intraoperative repositioning. Urology 78:357-364

6. Autorino R, Zargar H, Kaouk JH (2014) Robotic-assisted laparoscopic surgery: recent advances in urology. Fertil Steril 102:939-949

7. Rodriguez JF, Packiam VT, Boysen WR, Johnson SC, Smith ZL, Smith ND, Shalhav AL, Steinberg GD (2017) Utilization and outcomes of nephroureterectomy for upper tract urothelial carcinoma by surgical approach. J Endourol 31:661-665

8. Moher D, Liberati A, Tetzlaff J, Altman DG; PRISMA Group (2010) Preferred reporting items for systematic reviews and metaanalyses: the PRISMA statement. Int J Surg 8:336-341

9. Moher D, Shamseer L, Clarke M, Ghersi D, Liberati A, Petticrew M, Shekelle P, Stewart LA, PRISMA-P Group (2015) Preferred reporting items for systematic review and meta-analysis protocols (PRISMA-P) 2015 statement. Syst Rev 4:1. https://doi. org/10.1186/2046-4053-4-1

10. Howick J, Chalmers I, Glasziou P, Heneghan C, Liberati A, Moschetti I, Phillips B, Thornton H (2011) Explanation of the 2011 Oxford Centre for Evidence-Based Medicine (OCEBM)
Levels of Evidence (background document). Oxford Centre for Evidence- Based Medicine. https://www.cebm.net/index .aspx $? 0=5653$.

11. Wells GA, Shea B, O'Connell D, Robertson J, Peterson J, Welch V, Losos M, Tugwell P (2013) The Newcastle-Ottawa Scale (NOS) for assessing the quality of nonrandomized studies in meta-analyses. Ottawa Hospital Research Institute. http://www. ohri.ca/programs/clinical_epidemiology/oxford.asp. Accessed 6 June 2019

12. Jadad AR, Moore RA, Carroll D et al (1996) Assessing the quality of reports of randomized clinical trials: is blinding necessary? Control Clin Trials 17:1-12

13. Hozo SP, Djulbegovic B, Hozo I (2005) Estimating the mean and variance from the median, range, and the size of a sample. BMC Med Res Methodol 5:13. https://doi.org/10.1186/1471-2288-5-13

14. Ades AE, Lu G, Higgins JP (2005) The interpretation of randomeffects meta-analysis in decision models. Med Decis Making 25:646-654

15. Soria F, Shariat SF, Lerner SP, Fritsche HM, Rink M, Kassouf W, Spiess PE, Lotan Y, Ye D, Fernández MI, Kikuchi E, Chade DC, Babjuk M, Grollman AP, Thalmann GN (2017) Epidemiology, diagnosis, preoperative evaluation and prognostic assessment of upper-tract urothelial carcinoma (UTUC). World J Urol 35:379-387

16. Lee H, Kim HJ, Lee SE, Hong SK, Byun SS (2019) Comparison of oncological and perioperative outcomes of open, laparoscopic, and robotic nephroureterectomy approaches in patients with non-metastatic upper-tract urothelial carcinoma. PLOS ONE 14:e0210401. https://doi.org/10.1371/journal.pone.0210401

17. Lim SK, Shin TY, Kim KH, Han WK, Chung BH, Hong SJ, Choi YD, Rha KH (2014) Laparoendoscopic single-site (LESS) robot-assisted nephroureterectomy: comparison with conventional multiport technique in the management of upper urinary tract urothelial carcinoma. BJU Int 114:90-97

18. Melquist JJ, Redrow G, Delacroix S, Park A, Faria EE, Karam JA, Matin SF (2016) Comparison of single-docking robotic-assisted and traditional laparoscopy for retroperitoneal lymph node dissection during nephroureterectomy with bladder cuff excision for upper-tract urothelial carcinoma. Urology 87:216-223

19. Patel MN, Hemal AK (2018) Does advancing technology improve outcomes? comparison of the Da Vinci Standard/S/Si to the Xi robotic platforms during robotic nephroureterectomy. J Endourol 32:133-138. https://doi.org/10.1089/end.2017.0477

20. Argun OB, Mourmouris P, Tufek I, Tuna MB, Keskin S, Obek C, Kural AR (2016) Radical nephroureterectomy without patient or port repositioning using the Da Vinci Xi Robotic System: initial experience. Urology 92:136-139

21. Tinay I, Gelpi-Hammerschmidt F, Leow JJ, Allard CB, Rodriguez D, Wang Y, Chung BI, Chang SL (2016) Trends in utilisation, perioperative outcomes, and costs of nephroureterectomies in the management of upper tract urothelial carcinoma: a 10-year population-based analysis. BJU Int 117:954-960

22. Trudeau V, Gandaglia G, Shiffmann J, Popa I, Shariat SF, Montorsi F, Perrotte P, Trinh QD, Karakiewicz PI, Sun M (2014) Robot-assisted versus laparoscopic nephroureterectomy for uppertract urothelial cancer: A population-based assessment of costs and perioperative outcomes. Can Urol Assoc J 8:E695-701

23. Nazzani S, Bazinet A, Preisser F, Mazzone E, Tian Z, Mistretta FA, Shariat SF, Saad F, Zorn KC, Montanari E, Briganti A, Carmignani L, Karakiewicz PI (2019) Comparison of perioperative outcomes between open and minimally invasive nephroureterectomy: A population-based analysis. Int J Urol 26:487-492

24. Zargar H, Krishnan J, Autorino R, Akca O, Brandao LF, Laydner H, Samarasekera D, Ko O, Haber GP, Kaouk JH, Stein RJ (2014) Robotic nephroureterectomy: a simplified approach requiring no patient repositioning or robot redocking. Eur Urol 66:769-777 
25. Moschini M, Foerster B, Abufaraj M, Soria F, Seisen T, Roupret M, Colin P, De la Taille A, Peyronnet B, Bensalah K, Herout R, Wirth MP, Novotny V, Chlosta P, Bandini M, Montorsi F, Simone G, Gallucci M, Romeo G, Matsumoto K, Karakiewicz P, Briganti A, Shariat SF (2017) Trends of lymphadenectomy in upper tract urothelial carcinoma (UTUC) patients treated with radical nephroureterectomy. World J Urol 35:1541-1547

26. Nouralizadeh A, Tabatabaei S, Basiri A, Simforoosh N, Soleimani M, Javanmard B, Ansari A, Shemshaki H (2018) Comparison of open versus laparoscopic versus hand-assisted laparoscopic nephroureterectomy: a systematic review and meta-analysis. J Laparoendosc Adv Surg Tech A 28:656-681. https://doi.org/10.1089/ lap.2017.0662

27. Ha YS, Chung JW, Choi SH, Lee JN, Kim BS, Kim TH, Yoo ES, Kwon TG, Byun SS, Choi YD, Kang HW, Yun SJ, Kim WJ, Kim HT (2017) Impact of a bladder cuff excision during radical nephroureterectomy on cancer specific survival in patients with upper tract urothelial cancer in Korea a retrospective, multiinstitutional study. Minerva Urol Nefrol 69:466-474

28. Mistretta FA, Carrion DM, Nazzani S, Vásquez JL, Fiori C, De Cobelli, Porpiglia F, Esperto F (2019) Bladder recurrence of primary upper tract urinary carcinoma following nephroureterectomy, and risk of upper urinary tract recurrence after ureteral stent positioning in patients with primary bladder cancer. Minerva Urol Nefrol 71:191-200. 10.23736/S0393-2249.19.03311-3.

29. Peyronnet B, Seisen T, Dominguez-Escrig JL, Bruins HM, Yuan CY, Lam T, Maclennan S, Ndow J, Babjuk M, Comperat E,
ZigeunerR SRJ, Burger M, Mostafid H, van Rhijn BWG, Gontero P, Palou J, Shariat SF, Roupret M (2019) Oncological outcomes of laparoscopic nephroureterectomy versus open radical nephroureterectomy for upper tract urothelial carcinoma: an European Association of Urology Guidelines systematic review. Eur Urol Focus 5:205-223. https://doi.org/10.1016/j.euf.2017.10.003

30. Simone G, Papalia R, Guaglianone S, Ferriero M, Leonardo C, Forastiere E, Gallucci M (2009) Laparoscopic versus open nephroureterectomy: perioperative and oncologic outcomes from a randomised prospective study. Eur Urol 56:520-526. https://doi. org/10.1016/j.eururo.2009.06.013

31. Foerster B, D'Andrea D, Abufaraj M, Broenimann S, Karakiewicz PI, Rouprêt M, Gontero P, Lerner SP, Shariat SF, Soria F (2019) Endocavitary treatment for upper tract urothelial carcinoma: a meta-analysis of the current literature. Urol Oncol. https://doi. org/10.1016/j.urolonc.2019.02.004

32. Territo A, Foerster B, Shariat SF, Rouprêt M, Gaya JM, Palou J, Breda A (2018) Diagnosis and kidney-sparing treatments for upper tract urothelial carcinoma: state of the art. Minerva Urol Nefrol 70:242-251. https://doi.org/10.23736/S0393-2249.18.03058-8

Publisher's Note Springer Nature remains neutral with regard to jurisdictional claims in published maps and institutional affiliations.

\section{Affiliations}

\section{Alessandro Veccia ${ }^{1,2} \cdot$ Alessandro Antonelli ${ }^{2} \cdot$ Simone Francavilla ${ }^{2} \cdot$ Claudio Simeone $^{2} \cdot$ Georgi Guruli $^{1}$. Homayoun Zargar ${ }^{3}$. Sisto Perdoná ${ }^{4}$. Matteo Ferro ${ }^{5}$. Giuseppe Carrieri ${ }^{6}$. Lance J. Hampton ${ }^{1}$. Francesco Porpiglia ${ }^{7}$. Riccardo Autorino ${ }^{1}$}

1 Division of Urology, VCU Health System, VCU Medical Center, PO Box 980118, Richmond, VA 23298-0118, USA

2 Urology Unit, ASST Spedali Civili Hospital, Department of Medical and Surgical Specialties, Radiological Science, and Public Health, University of Brescia, Brescia, Italy

3 Department of Surgery, Department of Urology, University of Melbourne, Royal Melbourne Hospital, Melbourne, Australia

4 Uro-Gynecological Department, Fondazione "G. Pascale" IRCCS, Naples, Italy
5 Division of Urology, European Institute of Oncology, Milan, Italy

6 Urology and Renal Transplantation Unit, Department of Medical and Surgical Sciences, University of Foggia, Foggia, Italy

7 Division of Urology, San Luigi Gonzaga Hospital, Orbassano, Turin, Italy 